\title{
Y-2481
}

\section{PORTABLE DETECTOR FY 2000 TASK 4 COMPLETION REPORT}

Z. W. Bell, M. W. Moyer BWXT Y-12, L.L.C.

M. J. Paulus

Oak Ridge National Laboratory

February 2001

Prepared by the Y-12 National Security Complex Oak Ridge, Tennessee 37831-8169 managed by

BWXT Y-12, L.L.C. for the

U.S. DEPARTMENT OF ENERGY under contract

DE-AC05-00OR222800 


\section{DISCLAIMER}

This report was prepared as an account of work sponsored by an agency of the United States Government. Neither the United States Government nor any agency thereof, nor any of their employees, makes any warranty, express or implied, or assumes any legal liability or responsibility for the accuracy, completeness, or usefulness of any information, apparatus, product, or process disclosed, or represents that its use would not infringe privately owned rights. Reference herein to any specific commercial product, process, or service by trade name, trademark, manufacturer, or otherwise, does not necessarily constitute or imply its endorsement, recommendation, or favoring by the United States Government or any agency thereof. The views and opinions of authors expressed herein do not necessarily state or reflect those of the United States Government or any agency thereof. 
Portable Detector

FY2000 Task 4 Completion Report

Fabrication of Detector

Z. W. Bell, M. W. Moyer, BWXT Y-12, L.L.C

M. J. Paulus, Oak Ridge National Laboratory

\section{EXECUTIVE SUMMARY}

The fabrication of boron-covered crystal scintillation detectors is described. Bulk boron-loaded epoxy material was cast and cut into $0.5 \mathrm{~mm}$-thick wafers that were mounted on $\mathrm{CdWO}_{4}$ and $\mathrm{CsI}(\mathrm{Tl})$ crystals. The crystals were mounted on miniature photomultiplier tubes and gamma spectra were obtained with the detectors.

\section{FABRICATION OF BORON-LOADED MATERIAL}

Previous work has demonstrated that high boron content material could be fabricated from either sintered natural boron carbide or by mixing elemental boron powder into epoxy. Sintered natural boron carbide contains $0.34 \mathrm{~g} / \mathrm{cc}{ }^{10} \mathrm{~B}$ (because of the stoichiometry of boron carbide) while a mass of low viscosity epoxy was found to be able to take up an approximately equal mass of boron powder, resulting in up to $0.65 \mathrm{~g} / \mathrm{cc}$ boron. By using enriched boron powder instead of natural boron, material was realizable having a (thermal) neutron mean free path continuously adjustable down to $0.067 \mathrm{~mm}$. Because of the ease and speed with which boron-epoxy mixtures could be made, and the simplified processing to produce wafers, "ingots," cylinders, and annuli, these mixtures were used.

Low viscosity epoxies Master Bond EP30LV and Buehler Epothin were tested with -325 mesh natural boron powder to determine the maximum workable loading. Samples were prepared with $0,20,40,45$, and $50 \%$ by weight (boron weight as a percentage of the weight of epoxy plus boron), centrifuged to remove entrained air, and allowed to harden in $1 \mathrm{~cm}$ square plastic spectroscopy cuvettes. Mixtures of polyester resin and boron were also prepared, but it was found that the presence of metallic boron powder interfered with the polymerization of the plastic.

Although the viscosity of the uncured epoxy increased with the addition of boron powder, the increase posed no difficulties when working with the material until the boron fraction was in excess of $40 \%$. At $45 \%$, the Buehler material became extremely stiff and took on the consistency of dry dough. The Master Bond material remained workable to $50 \%$ boron fraction, but at that level, centrifugation at $900 \mathrm{Gs}$ failed to collapse all entrained air pockets. Further work was conducted with the Master Bond material.

Although centrifugation was found to be an effective method of removing entrained air, it was also found to segregate the suspended boron, probably by particle mass, and to cause a variation of boron density over the length of the ingots made in cuvetttes. Samples were examined both with ultrasound and computed tomography (CT). Ultrasound is most sensitive to variations in the speed of sound in materials and that sound is reflected and refracted at interfaces between materials of different mass density, Young's modulus, or acoustic velocity. In the case of epoxyboron mixtures, it is known that the speed of sound increases with increasing boron concentration, it would be expected that sound transmitted through or reflected from zones in the ingot with different acoustic properties would show variations in amplitude (relative to the incident amplitude) and transit time. CT, on the other hand, is most sensitive to variations in 
mass density and atomic number and highlights differences in x-ray absorption properties. Since the atomic numbers of boron and the constituents of epoxy are almost identical, CT is most sensitive to density variations.

Figure 1 shows ultrasound scans of samples containing 30\% boron and indicate the presence of three distinct zones. In the figure, the color scale uses the progression of colors of the rainbow (red, orange, yellow, green, blue, violet) to represent linearly increasing values. Thus, red represents a smaller value than orange. The samples are oriented such that the bottom of the sample is at the top of the figure. Figure 1a shows the amplitude of the ultrasound reflected from the back surface of the ingot (the sound pulse entered the ingot, traversed the 1-cm thickness, reflected from the back surface, and returned to the transducer). Since reflections are caused by changes in acoustic impedance, it can be inferred that there are three zones with different density in each. The boundaries between the zones are caused by destructive interference between the portions of the sound pulse scattered from each zone. Figure $1 \mathrm{~b}$ shows the time of flight of the sound pulse and indicates that the time to traverse the ingot decreases as one goes from the zone at the bottom of the figure to the zone at the top of the figure. Since it is known that the speed of sound through boron-epoxy mixtures increases with the boron concentration, these figures indicate increasing boron concentration toward the bottoms of the samples (top of the figure).

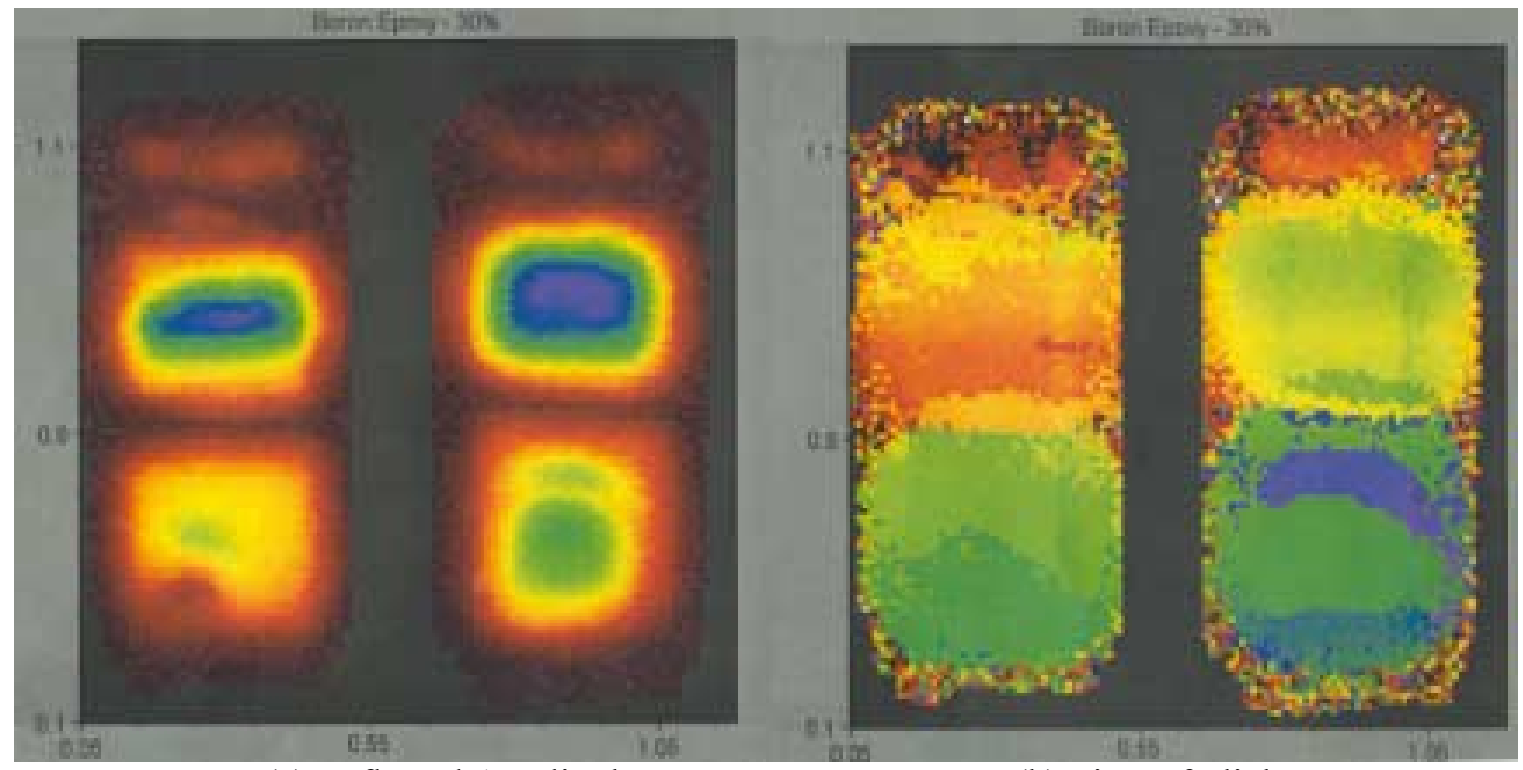

(a) Reflected Amplitude

(b) Time of Flight

Fig. 1. Ultrasound scans of $30 \%$ boron samples. Horizontal and vertical scales are in inches.

Figure 2 shows computed tomography (CT) scans of the same samples and corroborate the ultrasound results. The three zones are marked in the figure. Note that darker pixels imply less material and therefore lower density. 

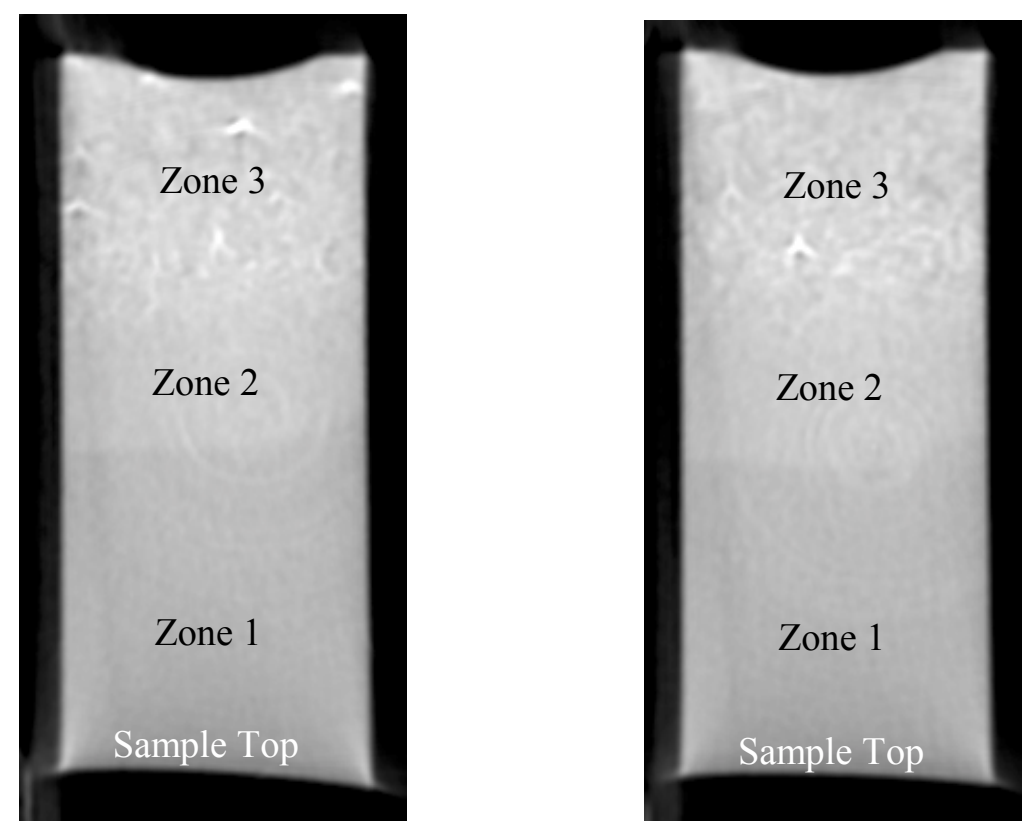

Fig. 2. CT scans of two $30 \%$ boron samples centrifuged 24 hours.

The process was then modified to allow air bubbles to rise to the surface of the cuvette under their own buoyancy. Boron, epoxy, and hardener were mixed together and stirred for one minute, with care being taken to scrape dry boron powder adhering to the side of the mixing cup into the mixture. The material was allowed to rest for ten minutes to permit air bubbles to rise to the surface. The mixture was then stirred gently (so as to entrain a minimum of air) for one minute to resuspend any settled powder and allowed to rest for five minutes. An additional stirring and rest period was followed by a gentle 1-minute stir, immediately after which the mixture was poured into one or more molds.

The working time of the Master Bond epoxy was approximately 1 hour, with complete cure at room temperature requiring 24 hours. Consequently, during the approximately 25 minutes of stirring and resting, the viscosity of the epoxy noticeably increased. Thus, boron suspended during the final stirring tended to remain in suspension during the next few hours while the epoxy set. Although the extra stirring resulted in some residual porosity from trapped air, no variation of boron concentration was detectable either by ultrasound or CT. Figures 3 and 4 show ultrasound and CT scans of $13 \%{ }^{10} \mathrm{~B}$ material fabricated by this process and the velocity profiles are seen to be essentially constant. The tops of the sample cuvettes are at the top of the figures. 


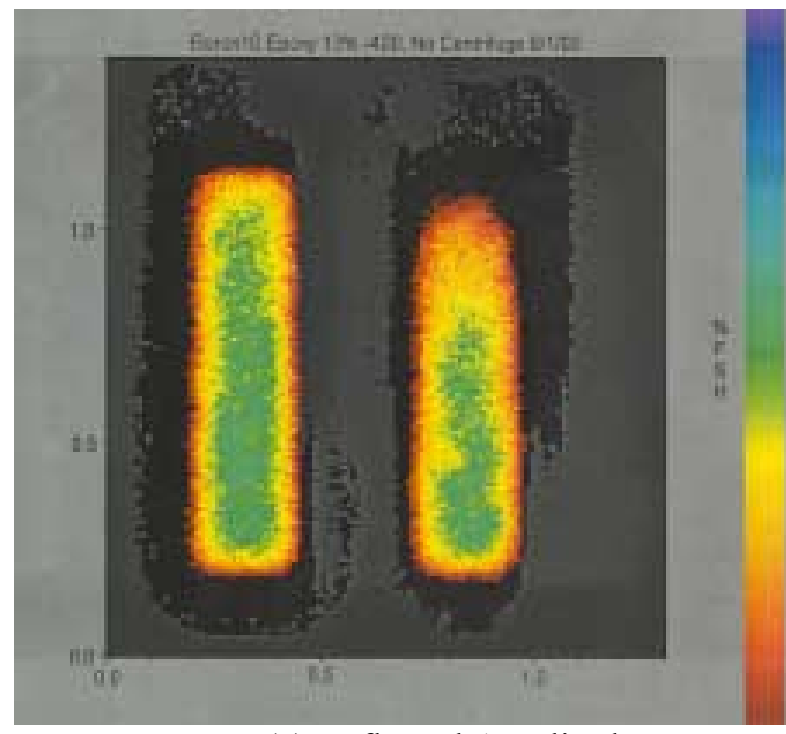

(a) Reflected Amplitude

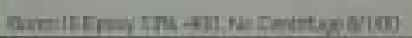

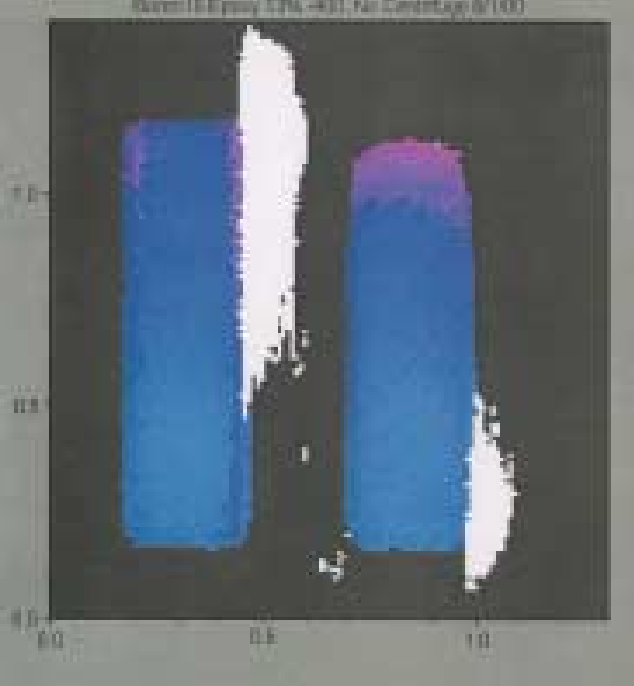

(b) Time of Flight

Fig. 3. Ultrasound scan of $13 \%$ boron loaded epoxy.
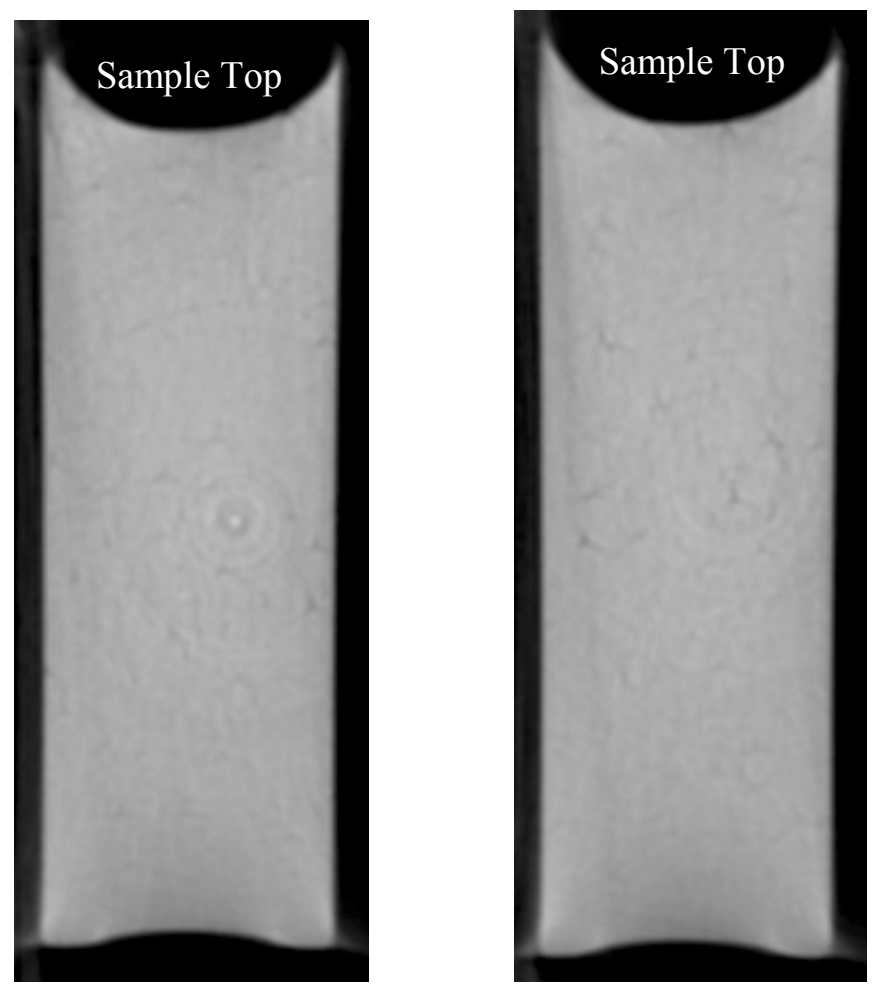

Fig. 4. CT images of $13 \%{ }^{10} \mathrm{~B}$ samples fabricated via the modified procedure.

The ${ }^{10} \mathrm{~B}$ fraction necessary to achieve $99 \%$ absorption of thermal neutrons in a thickness $\mathrm{t}$ can be computed from the following equations that relate the mass of boron, epoxy, and their densities:

$$
\begin{aligned}
& M_{B}+M_{E}=M \\
& V_{B}+V_{E}=V
\end{aligned}
$$




$$
\begin{aligned}
& \frac{M_{B}}{V_{B}}=\rho_{B} \\
& \frac{M_{E}}{V_{E}}=\rho_{E} \\
& \frac{M_{B}}{V}=\hat{\rho}_{B} \\
& \frac{\hat{\rho}_{B}}{10}(3838)(0.6022) t=5
\end{aligned}
$$

Equation 1 states that the mass of the mixture is the sum of the boron and epoxy masses. Equation 2 is a consequence of the immiscibility of epoxy and boron powder and states that the volume of the mixture is the sum of the volumes of the powder particles and the epoxy. Equations $3 \mathrm{a}$ and $3 \mathrm{~b}$ are the definitions of the density of solid boron (which is not the bulk density of boron powder), and cured epoxy. The latter was measured to be $1.18 \mathrm{~g} / \mathrm{cc}$; boron density was taken to be $2.35 \mathrm{~g} / \mathrm{cc}$. Equation $3 \mathrm{c}$ is the definition of the density of boron in the boron-epoxy mixture. Equation 4 states that the thickness, $\mathrm{t}$ (in $\mathrm{cm}$ ), of the wafer is five times the mean free path of thermal neutrons in the mixture.

Equations 2, 3a, 3b, and 3c can be manipulated to eliminate the volumes and obtain a relation between the boron and epoxy masses.

$$
M_{E}=M_{B} \rho_{E}\left(\frac{1}{\hat{\rho}_{B}}-\frac{1}{\rho_{B}}\right)
$$

Substituting (5) into (1) results in an expression, in terms of the bulk densities of the epoxy and boron, for the boron mass fraction necessary to achieve five thermal neutron mean free paths in a wafer of thickness $t$.

$$
\frac{M_{B}}{M}=\frac{1}{1+\rho_{E}\left(\frac{1}{\hat{\rho}_{B}}-\frac{1}{\rho_{B}}\right)}=\frac{1}{1+\rho_{E}\left(46.22 t-\frac{1}{\rho_{B}}\right)}
$$

To achieve $99 \%$ absorption in $0.5 \mathrm{~mm}$, a mixture boron density of $0.44 \mathrm{~g} / \mathrm{cc}$ is necessary. Equation 6 then predicts that the necessary boron fraction is approximately $32 \%$. For $99 \%$ absorption in $1.0 \mathrm{~mm}$, the necessary loading is approximately $15 \%$. A set of $15 \%$ and $32 \%{ }^{10} \mathrm{~B}$ epoxy rods were made so made was removed from the cuvettes and cut into $0.5 \mathrm{~mm}$ wafers with a diamond saw fitted with a wafering blade.

\section{FABRICATION OF DETECTORS}

Hamamatsu 5780 miniature photomultiplier modules were selected for this detector. This module has a TO- 8 photomultiplier tube, tube base, and high-voltage generator mounted in a package approximately $20 \mathrm{~mm}$ square by $60 \mathrm{~mm}$ long. The tube has current gain of approximately 300,000 and the Cockcroft-Walton high-voltage generator requires only the application of 11.5 to $15.5 \mathrm{VDC}$.

$\mathrm{CdWO}_{4}$ and $\mathrm{CsI}(\mathrm{Tl})$ crystals $6.3 \mathrm{~mm}$ square $(9 \mathrm{~mm}$ diagonal) by $20 \mathrm{~mm}$ long were procured from Saint-Gobain Crystals (formerly Bicron). Although rectangular crystals do not make the most efficient use of the $10 \mathrm{~mm}$ diameter circular photomultiplier window, this shape was necessary 
for $\mathrm{CdWO}_{4}$ because this material's crystal structure does not make it amenable to grinding to a cylinder. Although $\mathrm{CsI}(\mathrm{Tl})$ is easily ground into cylinders, a rectangular crystal was mounted so that direct comparisons between the two could be made.

The crystals were wrapped in white teflon tape on all but one of the $6.3 \mathrm{~mm}$ square faces. Bicron BC630 optical grease was applied to the bare faces and the crystals were pressed onto the photomultiplier windows. One $\mathrm{cm}$ square by $0.5 \mathrm{~mm}$ thick boron-epoxy wafers were trimmed to fit onto the five sides of the mounted crystals and held in place with vinyl electrical tape.

Aluminum foil was molded to the covered mounted crystal and black vinyl electrical tape was used to make a light-tight and mechanically sound assembly.

The detectors were exposed to gamma rays and neutrons. Figures 5 - 8 show the spectra obtained with the $\mathrm{CdWO}_{4}$ crystal from ${ }^{137} \mathrm{Cs},{ }^{241} \mathrm{Am},{ }^{133} \mathrm{Ba}$, and ${ }^{60} \mathrm{Co}$ placed opposite a 6.3 by $20 \mathrm{~mm}$ face and the $6.3 \mathrm{~mm}$ square face. All spectra, with the exception of ${ }^{60} \mathrm{Co}$, show the usual peaks when the source is placed opposite a long side; the ${ }^{60} \mathrm{Co}$ peaks are too energetic to produce a significant full energy peak in only $6.3 \mathrm{~mm}$ of crystal. When the sources are viewed through the square end, however, full energy peaks, including a weak peak from Co, are visible.

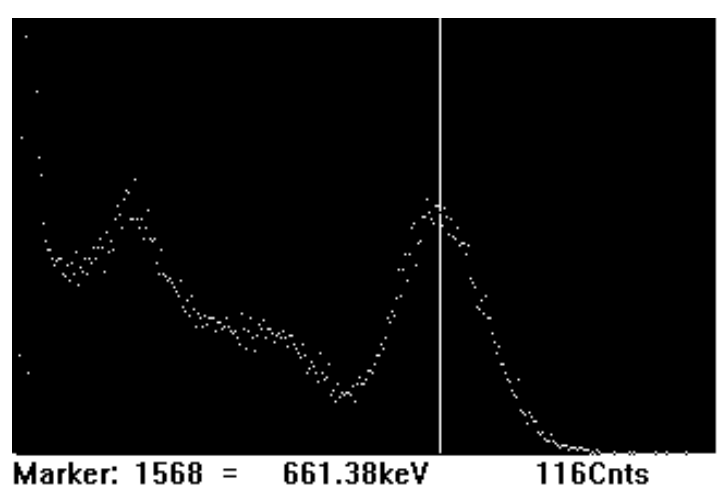

Fig. 5a. ${ }^{137}$ Cs opposite rectangular face.

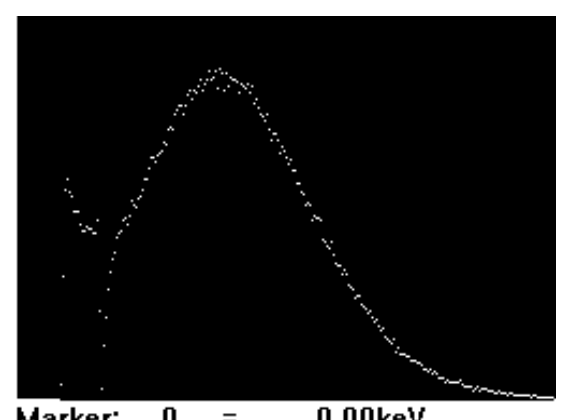

Marker: $0=0.00 \mathrm{keV}$

Fig. 6a. ${ }^{241}$ Am opposite rectangular face.

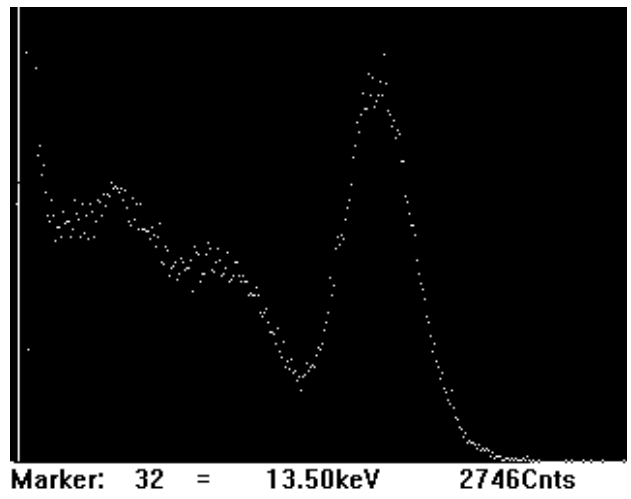

Fig. 5b. ${ }^{137}$ Cs opposite square end.

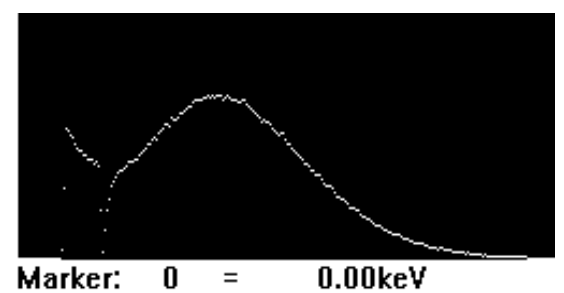

Fig. 6b. ${ }^{241} \mathrm{Am}$ opposite square end. 


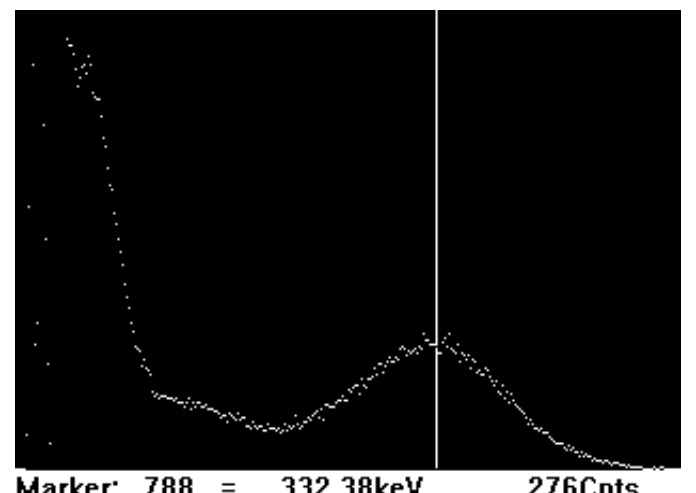

Fig. $7 \mathrm{a} .{ }^{133} \mathrm{Ba}$ opposite rectangular face.

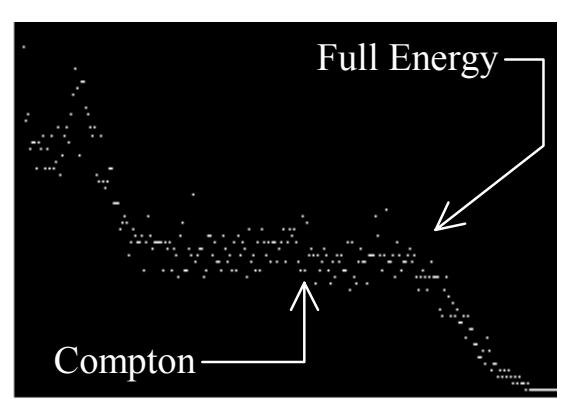

Marker: $0=0.00 \mathrm{keV}$

Fig. 8a. ${ }^{60} \mathrm{Co}$ opposite rectangular face.

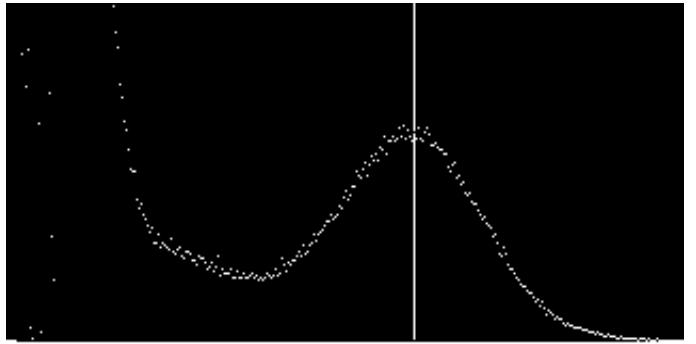

Marker: $764=322.25 \mathrm{keV} \quad 485 \mathrm{Cnts}$

Fig. 7 b. ${ }^{133} \mathrm{Ba}$ opposite square end.

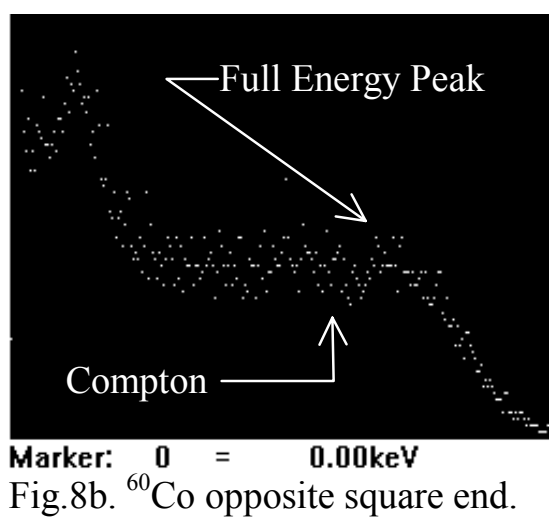

In Figs. 8a and $8 \mathrm{~b}$ the two cobalt lines are not resolved; a larger crystal is needed for that. The Compton edge is barely visible to the left of the full energy peaks.

Spectra obtained with the CsI(Tl) crystal were essentially identical to those in Figs. 5 - 8, with the exception that the $\mathrm{CsI}(\mathrm{Tl})$ crystal is brighter than the $\mathrm{CdWO}_{4}$. Consequently, although $\mathrm{CdWO}_{4}$ is denser than $\mathrm{CsI}(\mathrm{Tl})$ and should produce better photopeaks, the latter appears to be a better choice because of the higher intrinsic brightness of the crystal. The better counting statistics, peak shape, and resolution observed with the $\mathrm{CsI}(\mathrm{Tl})$ are expected to make spectrum analysis easier.

The $\mathrm{CdWO}_{4}$ detector was inserted into an Am-Li neutron source providing approximately 100 $\mathrm{n} / \mathrm{cm}^{2} / \mathrm{sec}$ thermal neutrons. Figures $9 \mathrm{a}$ and $9 \mathrm{~b}$ show the response of the detector, under otherwise identical conditions, with and without a $\mathrm{Cd}$ metal cover. The $478 \mathrm{keV}$ gamma ray peak due to the de-excitation of lithium from the ${ }^{10} \mathrm{~B}(\mathrm{n}, \alpha){ }^{*} \mathrm{Li}$ reaction is not present in Figure 9a, while it stands out against the Compton continuum caused by $2.22 \mathrm{MeV}$ hydrogen capture gammas, and tungsten capture gammas produced by the moderator and shielding within the source. 


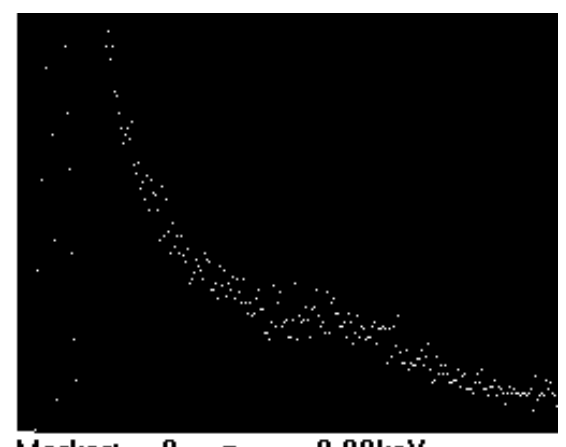

Marker: $0=0.00 \mathrm{keV}$

Fig. 9a. Detector covered with Cd metal.

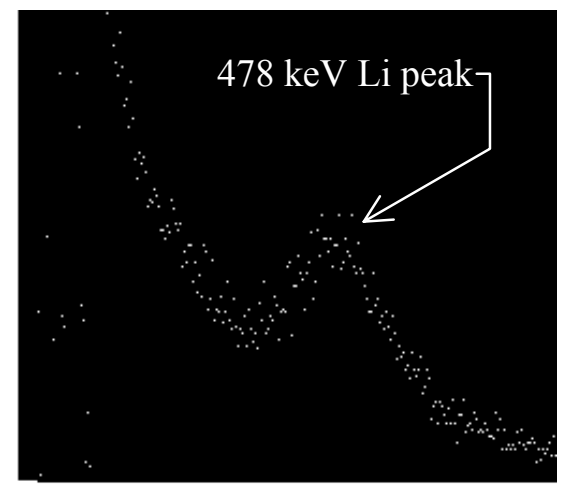

Marker: $0=0.00 \mathrm{keV}$

Fig. 9b. Response of detector to neutrons

\section{CONCLUSIONS}

The ability of these small detectors to produce spectra that can be analyzed to provide isotopic identification has been demonstrated. In addition, the detector can produce a signature indicating the presence of neutrons.

The same miniature size of these detectors that makes them attractive for hand-held portable use, may be a limiting factor in their efficiency. The small size of the scintillation crystals makes them not as efficient as larger $\mathrm{NaI}(\mathrm{Tl})$ crystals simply by virtue of significantly decreased sensitive volume and surface area. It may be worthwhile to consider slightly larger crystals (approximately $15 \mathrm{~mm}$ cubic $\mathrm{CdWO}_{4}$ ) mounted on rectangular photomultipliers in a detecting head connected to the electronics package by a signal cable. 
Distribution:

Z. W. Bell, 9723-24 MS 8084

M. W. Moyer, 9203-A MS 8084

V. M. Baylor, 1099COM MS 8260 (2)

Y-12 Central Files

Oak Ridge National Laboratory

M. J. Paulus, 3500 MS 6006 